\title{
Decentralized constrained optimal control of the multimachine power system stability improvement
}

\author{
Djibrine. Abakar', A. A. Abouelsoud ${ }^{2}$, Michael. J. Saulo ${ }^{3}$, S. Simiyu. Sitati ${ }^{4}$ \\ ${ }^{1}$ Department of Electrical Engineering, Pan African University, Institute for Basic Sciences, \\ Technology and Innovations (PAUISTI) Nairobi, Kenya \\ ${ }^{1}$ Department of Electrical Engineering, Polytechnic University of Mongo (UPM), Chad \\ ${ }^{2}$ Electronics and Electrical Communication Engineering, Cairo University (CU), Egypt \\ ${ }^{3}$ Department of Electrical Engineering, Technical University of Mombasa (TUM), Kenya \\ ${ }^{4}$ Department of Electrical \& Communications Engineering, Moi University (MU), Kenya
}

\begin{tabular}{l}
\hline \hline Article Info \\
\hline Article history: \\
Received Jul 23, 2019 \\
Revised Sep 24, 2019 \\
Accepted Oct 8, 2019
\end{tabular}

\section{Keywords:}

Decentralized control Multimachine power system Optimal control constrained feedback state

\begin{abstract}
This paper proposes a decentralized constrained optimal control of the multimachine power system stability. Today's power networks operate closer to their maximum limits. Alternative Current power grids are more vulnerable and subject to instability than ever before. A three machine power system and four machines, power system connected with a lossy transmission line, is investigated. The linearized dynamical equations of the multimachine power system are obtained near to an equilibrium point, and it can be stabilized by using a decentralized constrained controller based on optimal control. The feedback controller, which comprises independent control stations, receives the measurement data and influences the control input of the machine attached to it the subsystems. State feedback controller guarantees that the closed-loop system is stable and can guarantee the performance index. The designed controlled systems are based on the Algebraic Riccati Equations, and all its poles are in the closed left half-plane. Decentralized constrained optimal control of the multimachine power system is achieved through simulation results. The following results are proposed by improving power system stability.
\end{abstract}

Copyright $\odot 2020$ Institute of Advanced Engineering and Science. All rights reserved.

\section{Corresponding Author:}

Djibrine Abakar,

Department of Electrical and Electronic Engineering,

62000-00200 PAUISTI-JKUAT Nairobi, Kenya.

Email: djibrineabakar@hotmail.com

\section{INTRODUCTION}

Power systems stability problem has been enumerated and continues to receive a great deal of attention over the years. Stability of a power system is the ability of the power system to return to its normal conditions after being subjected to a disturbance, such as a system fault such as a transmission line outage for generator outage [1-2]. Supporting a power system with several machines and supplying continuously that is a motivating operating system in different scenarios could not lead to failure of the procedures and blackouts. A decentralized controller is a feedback controller, which consists of independent control stations, each of which receives the measurement data and influence the control input only from the attached subsystems [3]. The information flow from the plant through the controller in the feedback loop that is divided into a separated parallel flow through the control stations. Decentralization is an effective way to deal with complexity and cross changes in the interactions of large-scale dynamic systems. Although the optimal control concept has many advantages, it involves a complicated route in solving the problems of nonlinear systems.

Different methods have been used to improve decentralization of the multimachine in recent decades. $\mathrm{Xi}$ [4] designed a nonlinear decentralized controller used in Hamiltonian functions. The methods of the 
saturated steam valve and excitation controller in the power system. Ortega [5] Proposed transient stabilization of multimachine power systems with nontrivial transfer conductance based on Hamiltonian port and estimated the domain of attraction of a bona fide Lyapunov function. Li [6] proposed a nonlinear robust decentralized by using linear matrix inequalities approach to multimachine cases, based on suboptimal guaranteed stability, cost performance to be operated by different operating point fault location. Abouelsoud [7] proposed a stabilizing controller for a single machine power system with a nonzero conductance of transmission line, based on the observer to estimate the power angle of the system. The stability of the reduced-order observer and closed-loop of the system incorporating using the Lyapunov direct method is for a single machine.

Huang [8] designed a new model of excitation decentralized constrained controller of the multimachine power system based on the optimal feedback gain. Lee and Liaw [9] proposed the solution of inverse linear optimal control for constant disturbance using Algebraic Riccati Equations based on the linearquadratic regulator for an optimal feedback law and the relationships between the open-loop poles and the closed-loop poles that guarantee a positive regulator. Abouelsoud [10] proposed a global stabilization of linear time-invariant systems subject to state and control constraints based on the direct feedback controller to globally asymptotically stabilize a continuous-time linear time-invariant subjected to symmetrical polyhedral state and control constraints sets.

Elloumi [11] compared a decentralized control law to implement a multimachine power system. And it presented two aspects which are a linear decentralized control, and the gains depend on nonlinearity of the system and successive approximation approach to determine two-point boundary value. Hongshan and Xiaoming [12] proposed a fast excitation predictive control method for a multimachine power system based on a dynamic model and some inequality constraints on states, inputs, and outputs are considered in rolling optimization by using Gramian balanced reduction technique. Hosni and Selwa [13] investigated the problem of decentralized robust stabilizing control, based on direct Lyapunov methods and Kronecker reduction production notation. Tao [14] proposed an optimal control problem for large-scale systems with unknown parameters and dynamics. It uses a robust adaptive dynamic programming method and decentralized optimal control with unmatched uncertainties. The convergences of the robust adaptive dynamic programming algorithm and asymptotic stability of the closed-loop of the large-scale system are studied rigorously.

This paper proposed an improvement in the multimachine power system using decentralized constrained optimal control. Transient stability assesses using parameter space has been limited by the number of machines and stability are bounded because of size and dimension. Power system stabilizer and genetic algorithm have applied to a single machine infinite bus, which complicated for the multimachine system. The difficulties arise that use a random variation and selection of minimizing estimate error and time consumed for the artificial immune system and evolutionary programming [23-25].

\section{MODEL AND PROBLEM FORMULATION}

The paper considers the multimachine power systems consisting of generators interconnected through a transmission line which is assumed as a lossy. The dynamics of the $\mathrm{i}^{\text {th }}$ generators is presented by $[5,15-16]$.

The classical three-dimensional flux decay model presents the dynamic of the $\mathrm{i}^{\text {th }}$ generator with excitation.

$$
\begin{aligned}
& \dot{\delta}_{i}=\omega_{i} \\
& \dot{\omega}_{i}=-\frac{D_{m i}}{M_{i}}+\frac{\omega_{0}}{M_{i}}\left(P_{m i}-P_{e i}\right) \\
& \dot{E}_{i}=\frac{1}{\tau_{i}}\left(-E_{q i}+E_{f i}+V_{i}\right), i \in \bar{N}:=\{1, \ldots \ldots \ldots \ldots \ldots ., N\}
\end{aligned}
$$

Where the states variables of this subsystem are the rotor angle $\boldsymbol{\delta}_{\mathbf{i}} \in \mathbb{\Gamma}:=[\mathbf{0 , 2 \pi})$, rad, the speed deviation $\boldsymbol{\omega}_{\mathbf{i}} \in$ $\mathbb{R}$ in a rad/sec and the generator quadrature internal voltage $\mathbf{E}_{\mathbf{i}} \in \mathbb{R}_{+}$in p.u. $\mathrm{D}_{\mathrm{mi}}$ is the damping constant in pu. $M_{i}=2 H$, where $H$ is the inertia constants in seconds, $P_{m i}$ is the constants mechanical input power and $P_{e i}$ is the electromagnetic power, both in pu (per units), $\boldsymbol{\tau}_{\mathbf{i}}$ is the direct axis transient short circuit time constant. The voltages $\mathrm{E}_{\mathrm{qi}}, \mathrm{E}_{\mathrm{fi}}$ and $v_{\mathrm{i}}$, expressed in $\mathrm{pu}$ are the electromotive force in the quadrature axis, the control constant voltage component applied to the field winding and the control voltage input, respectively.

The active $\mathrm{P}_{\mathrm{ei}}$ and reactive $\mathrm{Q}_{\mathrm{ei}}$ power, as well as the voltage Eqi, are defined as: 


$$
\begin{aligned}
& P_{e i}=E_{i} I_{q i} \\
& Q_{e i}=E_{i} I_{d i} \\
& E_{q i}=E_{i}+\left(x_{d i}-x_{d i}^{\prime}\right) I_{d i}=x_{a d i} I_{f i}
\end{aligned}
$$

Where $\mathrm{I}_{\mathrm{qi}}$ and $\mathrm{I}_{\mathrm{di}}$ are the quadratures and direct axis currents, $\mathrm{x}_{\mathrm{di}}$ is the direct axis reactance and $\mathrm{x}$ ' di the direct axis transient reactance, where it says that $\mathrm{x}_{\mathrm{di}}>\mathrm{X}^{\prime}{ }_{\mathrm{di}} \mathrm{I}_{\mathrm{fi}}$ is the field winding current and $\mathrm{x}_{\text {adi }}$ is the mutual reactance between the excitation and stator coils. All the latter quantities are expressed in p.u. System.

The currents give the interconnection between machines.

$$
\begin{aligned}
I_{q i} & =\sum_{j=1}^{N} E_{j}\left(G_{m i j} \cos \delta_{i j}+B_{m i j} \sin \delta_{i j}\right) \\
I_{d i} & =\sum_{j=1}^{N} E_{j}\left(G_{m i j} \sin \delta_{i j}-B_{m i j} \cos \delta_{i j}\right)
\end{aligned}
$$

Where $\delta_{\mathrm{ij}}=\delta_{\mathrm{i}}-\delta_{\mathrm{j}}$, Gmij is the conductance and $\mathrm{B}_{\mathrm{mij}}$ is the susceptance both in pu resulting from the computation of the network admittance matrix. Developing the sums when $j=i$ and recalling the identities.

The network admittance through conductance and susceptance reads:

$$
\begin{aligned}
& Y_{i j}=\sqrt{G^{2}{ }_{m i j}+B^{2}}{ }_{m i j} \\
& \alpha_{i j}=\arctan \left(\frac{G_{m i j}}{B_{m i j}}\right) \\
& G_{m i j} \cos \delta_{i j}+B_{m i j} \sin \delta_{i j}=Y_{i j} \sin \left(\delta_{i j}+\alpha_{i j}\right) \\
& G_{m i j} \sin \delta_{i j}-B_{m i j} \cos \delta_{i j}=-Y_{i j} \cos \left(\delta_{i j}+\alpha_{i j}\right)
\end{aligned}
$$

Yields the current in quadratic and direct axis:

$$
\begin{aligned}
& I_{q i}=G_{m i i} E_{i}+\sum_{j=1}^{N} E_{j} Y_{i j} \sin \left(\delta_{i j}+\alpha_{i j}\right) \\
& I_{d i}=-B_{m i i} E_{i}-\sum_{j=1}^{N} E_{j} Y_{i j} \cos \left(\delta_{i j}+\alpha_{i j}\right)
\end{aligned}
$$

Finally, combining (1) and (4) results in the well-known compact form is the dynamic equation of the third-order model $\left(\dot{\delta}_{l}, \dot{\omega}_{l}, \dot{E}_{l}\right)$ which becomes:

$$
\begin{aligned}
& \dot{\delta}_{i}=\omega_{i} \\
& \dot{\omega}_{i}=-D_{i} \omega_{i}+P_{i}-G_{i i} E_{i}^{2}-d_{i} E_{i} \sum_{j=1, j \neq i}^{N} E_{j} Y_{i j} \sin \left(\delta_{i j}+\alpha_{i j}\right) \\
& \dot{E}_{i}=-a_{i} E_{i}+b_{i} \sum_{j=1, j \neq i}^{N} E_{j} Y_{i j} \cos \left(\delta_{i j}+\alpha_{i j}\right)+\frac{1}{\tau_{i}}\left(E_{f i}+v_{i}\right)
\end{aligned}
$$

With the positive constants:

$$
\begin{aligned}
& D_{i}=\frac{D_{m i}}{M_{i}}, P_{i}=d_{i} P_{m i}, G_{i i}=d_{i} G_{m i i}, d_{i}=\frac{\omega_{0}}{M_{i}} \\
& a_{i}=\frac{1}{\tau_{i}}\left(1-\left(x_{d i}-x_{d i}^{\prime}\right) B_{m i i}\right), b_{i}=\frac{1}{\tau_{i}}\left(x_{d i}-x_{d i}^{\prime}\right)
\end{aligned}
$$

Similarly, it observes that, $a_{i,}, b i>0 a_{i j}=\alpha_{i j}$, and that if $M_{i}=M_{j}, Y_{i j}=Y_{j i}$

It assumes that the model with $\mathrm{u}_{\mathrm{i}}=0$ has stabled the equilibrium points $\left[\delta_{i^{*}}, 0, E_{i^{*}}\right]$ and it finds the control law $\mathrm{u}_{\mathrm{i}}$ such that it operates in closed-loop. 


\section{STABILIZATION WITH STATE CONSTRAINTS}

Considering a continuous-time Linear Time-Invariant system as follows:

$$
\dot{x}(t)=A x(t)+B u(t)
$$

$x \in \mathbb{R}^{n}, u \in \mathbb{R}^{m},(A, B)$ it is a controllable pair and symmetric constraint state and control of sets.

$$
\begin{aligned}
& S_{x}=\left\{x \in \mathbb{R}^{n}:-\omega_{x} \leq G_{x} x \leq \omega_{x}\right\} \\
& S_{u}=\left\{u \in \mathbb{R}^{n}:-\omega_{u} \leq G_{u} x \leq \omega_{u}\right\}
\end{aligned}
$$

$G_{x} \in \mathbb{R}^{s 1 \times n}, E_{u} \in \mathbb{R}^{r 1 \times n}$ Are both full row rank, it considers the problem of designing a state feedback controller [10, 17, 21, 22].

By scaling, let us make $\omega_{x}=1$ and $\omega_{u}=\overline{1}$

$S_{x}=\left\{x \in \mathbb{R}^{n}:-\overline{1} \leq G_{x} x \leq \overline{1}\right\}$

$S_{u}=\left\{u \in \mathbb{R}^{m}:-\overline{1} \leq E_{u} x \leq \overline{1}\right\}$

Let

$G=\left[\begin{array}{c}G_{x} \\ 0\end{array}\right], E=\left(\begin{array}{c}0 \\ E_{u}\end{array}\right)$

$u=K_{x}$ then the state and control constraints become

$-\overline{1} \leq\left(\begin{array}{c}G \\ E K\end{array}\right) x \leq \overline{1}$ Or $-\overline{1} \leq G_{x}+E_{u} \leq \overline{1}$

Assume that the invariant zeros of the system (A, B, G, and E) are in the open left-half plane.

$$
u(t)=-K x(t)
$$

$K$ Is gain, such that the closed-loop system becomes:

$$
\dot{x}(t)=A_{c} x(t)
$$

Where $A_{c}=A-B K$ is asymptotically stable, and both state control constraints (8), (9) are satisfied. This implies that the state $S_{x}$ is $A_{c}$ invariant.

\subsection{Simulation Results}

The motion equation of the three-machine power system can be described by:

$$
\begin{aligned}
& \dot{\delta}_{1}=\omega_{1} \\
& \dot{\omega}_{1}=-D_{1} \omega_{1}+P_{1}-G_{11} E_{1}^{2}-Y E_{1} E_{2} \sin \left(\delta_{1}-\delta_{2}+\alpha\right) \\
& \dot{E}_{1}=-a_{1} E_{1}+b_{1} E_{2} \cos \left(\delta_{1}-\delta_{2}+\alpha\right)+E_{f 1}+u_{1} \\
& \dot{\delta}_{2}=\omega_{2} \\
& \dot{\omega}_{2}=-D_{2} \omega_{2}+P_{2}-G_{22} E_{2}^{2}-Y E_{1} E_{2} \sin \left(\delta_{1}-\delta_{2}-\alpha\right) \\
& \dot{E}_{2}=-a_{2} E_{2}+b_{2} E_{1} \cos \left(\delta_{2}-\delta_{1}+\alpha\right)+E_{f 2}+u_{2} \\
& \dot{\delta}_{3}=\omega_{3} \\
& \dot{\omega}_{3}=-D_{3} \omega_{3}+P_{3}-G_{33} E_{3}^{2}-Y E_{1} E_{3} \sin \left(\delta_{1}-\delta_{3}-\alpha\right) \\
& \dot{E}_{3}=-a_{3} E_{3}+b_{3} E_{1} \cos \left(\delta_{3}-\delta_{1}+\alpha\right)+E_{f 3}+u_{3}
\end{aligned}
$$

During the steady-state, all these parameters become as mentioned below to determine the equilibrium points: $\omega_{1}=0 ; \omega_{2}=0 ; \omega_{3}=0 ; u_{1}=0 ; u_{2}=0 ; u_{3}=0 ; \alpha=0$; $\delta_{10}, \delta_{20}, \delta_{30 \text { and }} E_{10}, E_{20}, E_{30}$ to be known and then; 
$f\left(x_{0}\right)=0$; as shown in (12) becomes:

$$
\begin{aligned}
& \left\{\begin{array}{l}
P_{1}-G_{11} E_{10}^{2}-Y E_{10} E_{20} \sin \left(\delta_{10}-\delta_{20}+\alpha\right)=0 \\
-a_{1} E_{10}+b_{1} E_{20} \cos \left(\delta_{10}-\delta_{20}+\alpha\right)+E_{f 1}=0 \\
P_{2}-G_{22} E_{20}^{2}-Y E_{10} E_{20} \sin \left(\delta_{10}-\delta_{20}+\alpha\right)=0 \\
-a_{2} E_{20}+b_{2} E_{20} \cos \left(\delta_{20}-\delta_{10}+\alpha\right)+E_{f 2}=0 \\
P_{3}-G_{33} E_{30}^{2}-Y E_{10} E_{30} \sin \left(\delta_{10}-\delta_{30}+\alpha\right)=0 \\
-a_{3} E_{30}+b_{3} E_{10} \cos \left(\delta_{30}-\delta_{10}+\alpha\right)+E_{f 3}=0
\end{array}\right. \\
& \sin \left(\delta_{10}-\delta_{20}+\alpha\right)=\frac{P_{1}-G_{11} E_{10}^{2}}{Y E_{10} E_{20}} \\
& \cos \left(\delta_{10}-\delta_{20}+\alpha\right)=\frac{a_{1} E_{10}-E_{f 1}}{b_{1} E_{20}} \\
& \tan \left(\delta_{30}-\delta_{10}+\alpha\right)=\frac{b_{3} P_{3}-b_{3} G_{33} E_{30}^{2}}{Y_{3} a_{3} E_{30}^{2}-Y_{3} E_{30} E_{f 3}} \\
& \tan \left(\delta_{40}-\delta_{10}+\alpha\right)=\frac{\left(G_{44} E_{40}^{2}-P_{4}\right) b_{4}}{Y_{4} E_{40}\left(a_{4} E_{40}-E_{f 4}\right)} \\
& \cos \left(\delta_{40}-\delta_{10}+\alpha\right)=\frac{Y_{4} E_{40} E_{f 4} \tan \left(\delta_{40}-\delta_{10}+\alpha\right)-b_{4} P_{4}}{E_{40}\left(a_{4} Y_{4} \tan \left(\delta_{40}-\delta_{10}+\alpha\right)-b_{4} G_{44}\right)} \\
& \sin \left(\delta_{10}-\delta_{40}-\alpha\right)=\frac{P_{4}-P_{2}+G_{11} E_{10}^{2}}{G_{41} E_{10}} \\
& E_{30}=\frac{b_{2} E_{40}}{a_{2} * b_{1}}\left[a_{1} * E_{10}-E_{f 1}\right]+\frac{E_{f 2}}{a_{2}} \\
& b_{3} P_{3}+Y_{3} E_{30} \tan \left(\delta_{30}-\delta_{10}+\alpha\right) E_{f 3} \\
& E_{30}\left[Y_{3} \tan \left(\delta_{30}-\delta_{10}+\alpha\right)+b_{3} G_{33}\right]
\end{aligned}
$$

Considering a power system network represented by four machines connected to a transmission line is lossy, as shown: 


$$
\begin{aligned}
& \dot{\delta}_{1}=\omega_{1} \\
& \dot{\omega}_{1}=-D_{1} \omega_{1}+P_{1}-G_{11} E_{1}^{2}-Y E_{1} E_{2} \sin \left(\delta_{1}-\delta_{2}+\alpha\right) \\
& \dot{E_{1}}=-a_{1} E_{1}+b_{1} E_{2} \cos \left(\delta_{1}-\delta_{2}+\alpha\right)+E_{f 1}+u_{1} \\
& \dot{\delta_{2}}=\omega_{2} \\
& \dot{\omega}_{2}=-D_{2} \omega_{2}+P_{2}-G_{22} E_{2}^{2}-Y E_{1} E_{2} \sin \left(\delta_{1}-\delta_{2}-\alpha\right) \\
& \dot{E}_{2}=-a_{2} E_{2}+b_{2} E_{1} \cos \left(\delta_{2}-\delta_{1}+\alpha\right)+E_{f 2}+u_{2} \\
& \dot{\delta}_{3}=\omega_{3} \\
& \dot{\omega}_{3}=-D_{3} \omega_{3}+P_{3}-G_{33} E_{3}^{2}-Y E_{1} E_{3} \sin \left(\delta_{1}-\delta_{3}-\alpha\right) \\
& \dot{E}_{3}=-a_{3} E_{3}+b_{3} E_{1} \cos \left(\delta_{3}-\delta_{1}+\alpha\right)+E_{f 3}+u_{3} \\
& \dot{\delta}_{4}=\omega_{4} \\
& \dot{\omega}_{4}=-D_{4} \omega_{4}+P_{4}-G_{44} E_{4}^{2}-Y E_{1} E_{4} \sin \left(\delta_{1}-\delta_{4}+\alpha\right) \\
& \dot{E}_{4}=-a_{4} E_{4}+b_{4} E_{1} \cos \left(\delta_{4}-\delta_{1}+\alpha\right)+E_{f 4}+u_{4}
\end{aligned}
$$

It determines the equilibrium points by assuming: $\omega_{1}=0 ; \omega_{2}=0 ; \omega_{3}=0 ; \omega_{4}=0 ; u_{1}=0 ; u_{2}=0 ; u_{3}=0$ $; u_{4}=0$; The calculation of the parameters is: $\delta_{10} ; \delta_{20} ; \delta_{30} ; \delta_{40} ; E_{10} ; E_{20} ; E_{30} ; E_{40}$

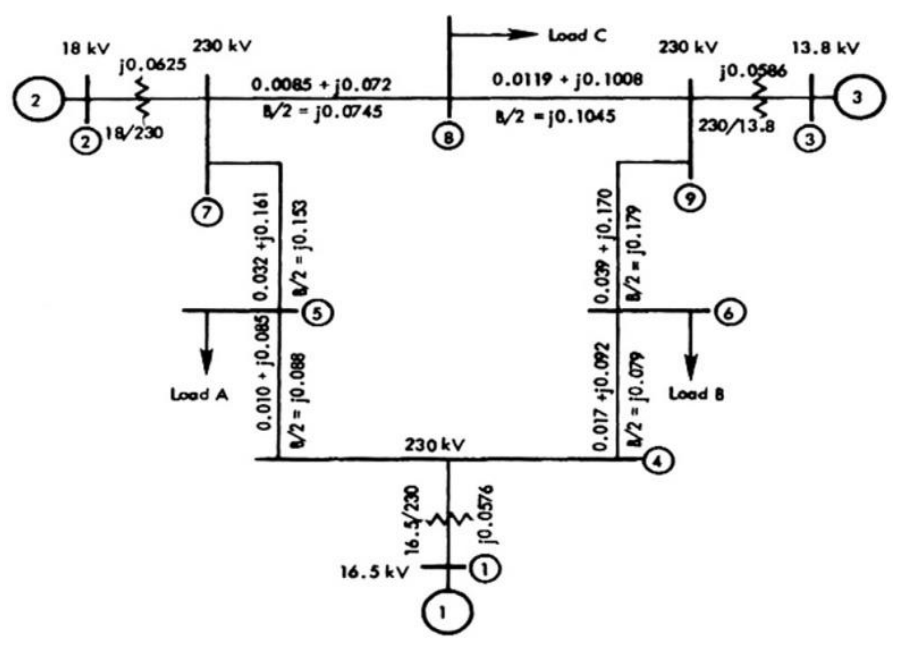

Figure 1. Three machines power systems [1]

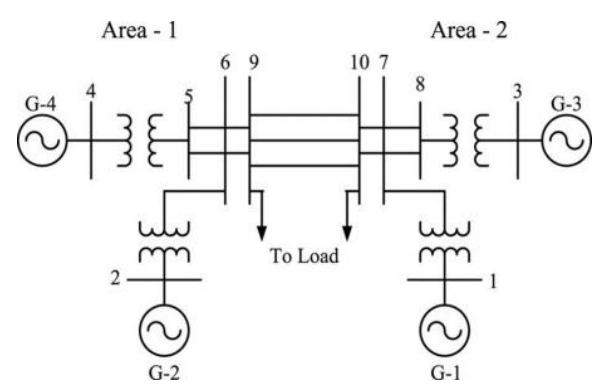

Figure 2. Four machines power systems [18-20] 


\section{RESULTS AND DISCUSSION}

These results are compared and discussed in the numerical procedure for the multimachine power system. Decentralized constrained controller not only stabilizes the power system but also achieves an optimal solution for overall subsystems. The numerical simulation of the three-machine power system and the fourmachine power system indicated the results.

\subsection{Three Machine System}

The simulations carried out on the three machines, a power system with a lossy transmission line and decentralized constrained controller is solved. A three-phase fault occurs at 1.5 seconds on the transmission line between bus 7 and 8 nearest to bus 8 in Figure 1. The fault is cleared by opening the circuit breakers both at the ends of the line. It makes over 2 seconds. During the steady-state, the conventional control system with: $u_{1}=0 ; u_{2}=0 ; u_{3}=0$, which cannot maintain the stability of the fault is cleared in 2.2 seconds after fault occurrences.

The optimal control decentralized constrained, and they give the feedback state gain matrix $K$ time response of the rotor angle; the rotor speed and the excitation system of the machines $\delta_{1} ; \delta_{2} ; \delta_{3} ; \omega_{1} ; \omega_{2} ; \omega_{3}$ $; E_{1} ; E_{2} ; E_{3}$ are illustrated in the Figures 3 in Figure 11 which show the responses of the multimachine system. Those figures show the advantage of the proposed control scheme. The simulation of the proposed control structure of the system under identical conditions leads to the following observations. It plots the results that show the rotor angle versus time, the rotor speed versus time, and the excitation system versus time are shown in Figure 3 to Figure 11 for each machine systems.

This control technique involves several independent local controllers decentralized through subsystem coordination. It considers a local feedback controller communicating among subsystem with constraint bounded. This advantage of the decentralized controller is reliable and enlargeable for each subsystem seems to achieve its objective. The decision is taken locally, and negotiation can take place in different actuators. They usually have different goals that are applied for three machine system and four machine system.

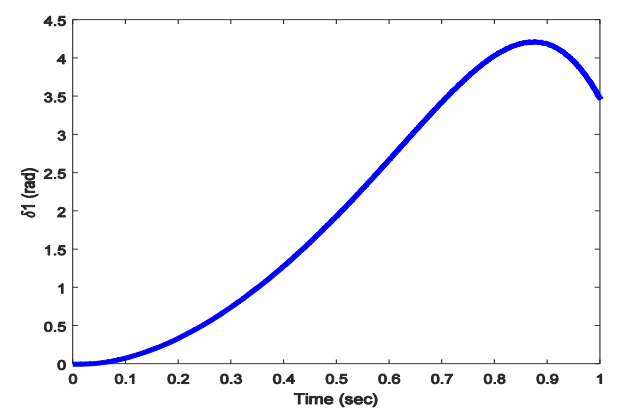

Figure 3. Rotor angle of machine \#1

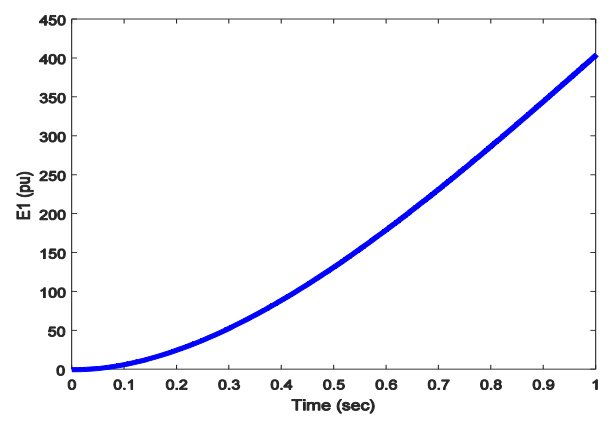

Figure 5. Excitation system of machine \#1

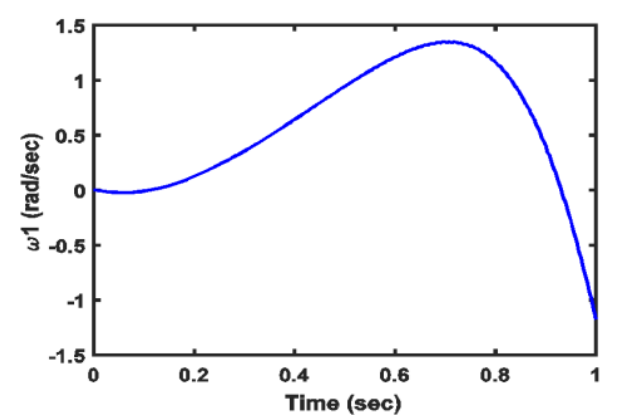

Figure 4. The rotor speed of machine \#1

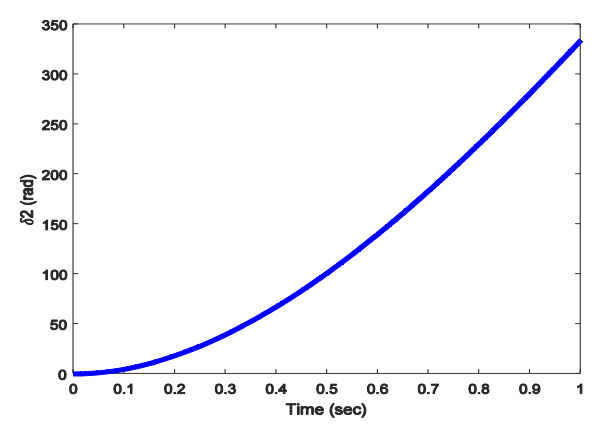

Figure 6. Rotor angle of machine \#2 


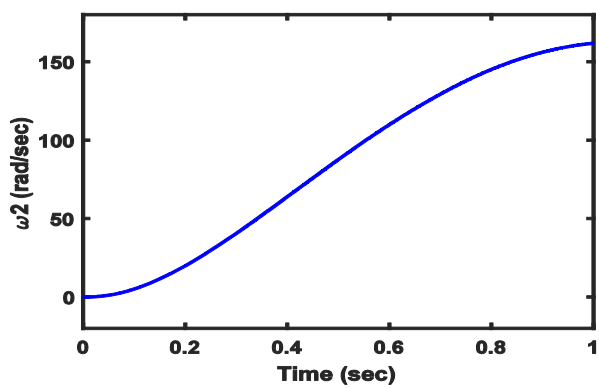

Figure 7. The rotor speed of machine \#2

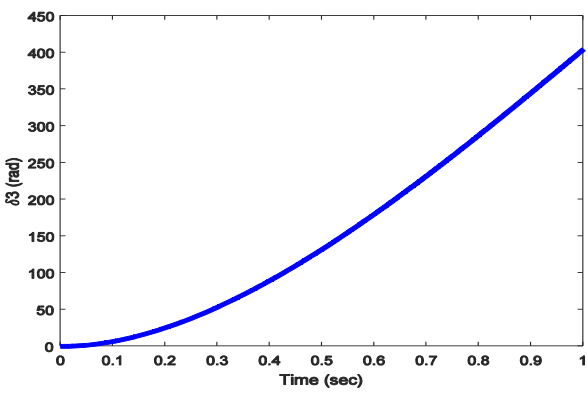

Figure 9. Rotor angle of machine \#3

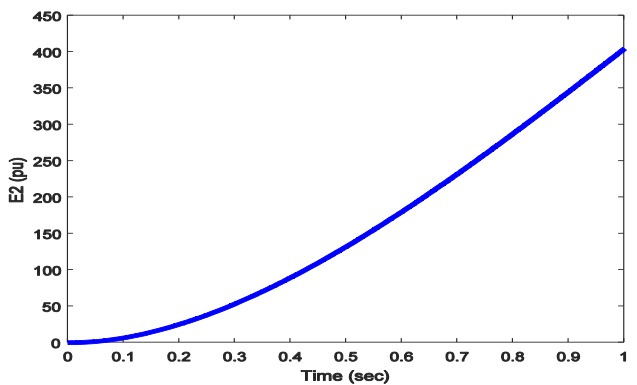

Figure 8. Excitation system of machine \#2

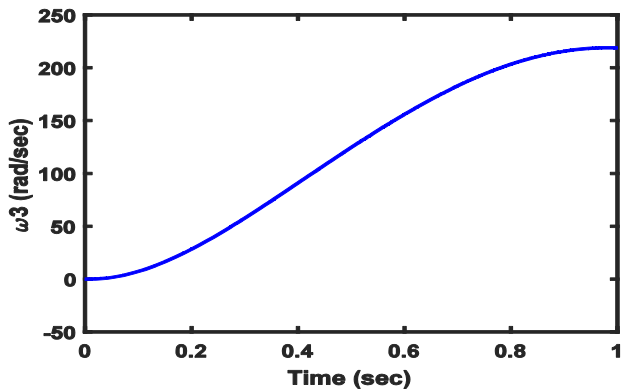

Figure 10. The rotor speed of machine \#3

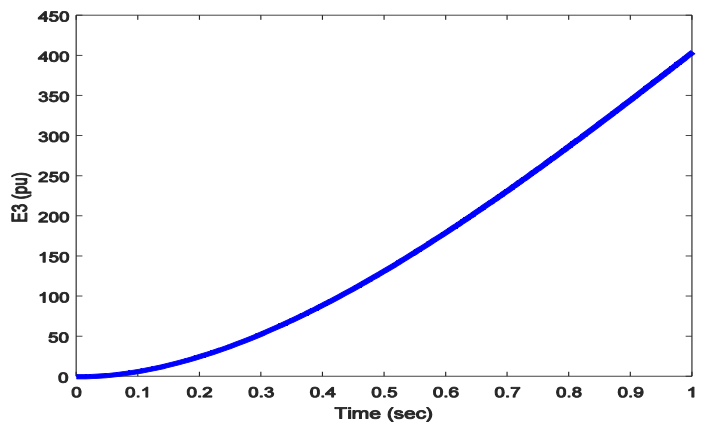

Figure 11. Excitation system of machine \#3

The analysis assumes that when there are no losses, the conductance is null. The equilibrium points at the steady-state after the fault is: $\left(\delta_{1 *}, \omega_{1 *}, E_{1 *}, \delta_{2 *}, \omega_{2 *}, E_{2 *}, \delta_{3^{*}}, \omega_{3 *}, E_{3 *}\right)=(0.03965,0,1.0566,0.344,0,1.0502,0.2299,0,1.0170)$ which verifies the conditions of the three machine power system in the dynamical model. The rotor angle of all the machines shown in the Figures 3, 6 and 9, are increasing during the fault occurrence and it regains to the normal state after clearing the fault between 0.7 seconds to 0.9 seconds, and it converges to the normal state. The rotor speed of the system in the Figures 4, 7 and 11, is in the steady-state and the increase after introducing faults at 0.7 seconds to 1 second and it regains to the normal state after the fault occurred. Voltage increased till 1 second and restore to the maximum level after the fault is cleared at 1 second to the normal state.

\subsection{Four Machines System}

This subsection presents simulations of the four-machine system in Figure 2. In this case, the disturbance is a three-phase bolted fault are applied in 0.5 seconds in the bus 9 . This fault is cleared by tripping line 1 . The evolution of the rotor angle, the rotor speed, and the excitation system of the generator are observed and detailed. Finally, graphs are shaped and presented. 
That modifies the topology of the network and consequently induces changes in the equilibrium points, as identified: $\left(\delta_{1 *}, \omega_{1 *}, E_{1 *}, \delta_{2 *}, \omega_{2 *}, E_{2 *}, \delta_{3 *}, \omega_{3 *}, E_{3 *}, \delta_{4 *}, \omega_{4 *}, E_{4 *}\right)=(0.6105,0,1.0397,0.8039,0,1.16,0.6105,0,1.0397,0.8039,0,1.16)$ and it verifies the conditions of the four machines power systems as connected.

The rotor angle of the machines is presented in Figures 12,15, 18 and 21, are stable during the steadystate and increase during the fault and stabilized at one second. The rotor speed of the machines is shown in Figures 13, 16, 19 and 22, are increasing up to 0.7 seconds, and it sustained at one second during the fault. The excitation system of the machines is depicted in the Figures 14, 17, 20 and 23, are decreased during from 0 seconds to -0.5 seconds and to stabilize during 10 milliseconds at overall and so far, to regain at one second to the normal state.

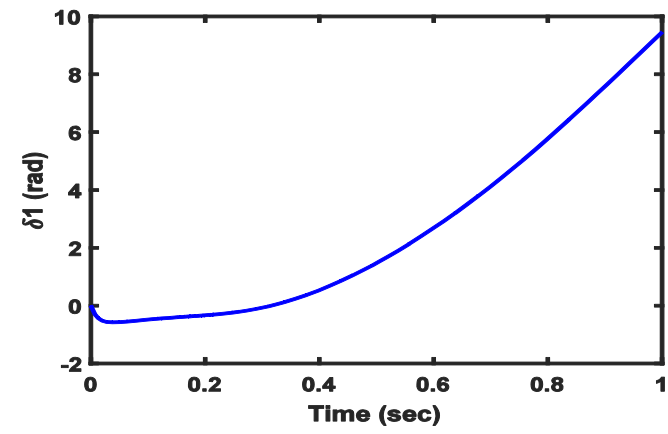

Figure 12. Rotor angle of machine \#1

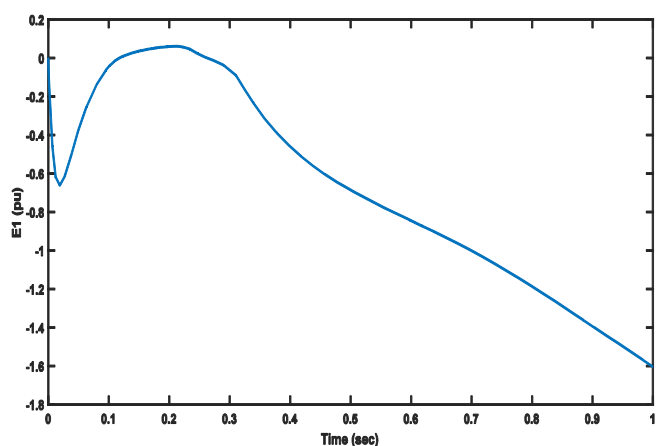

Figure 14. Excitation system of machine \#1

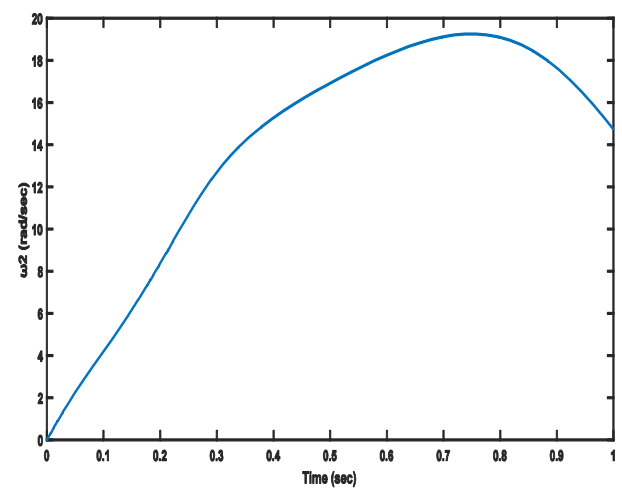

Figure 16. The rotor speed of machine \#2

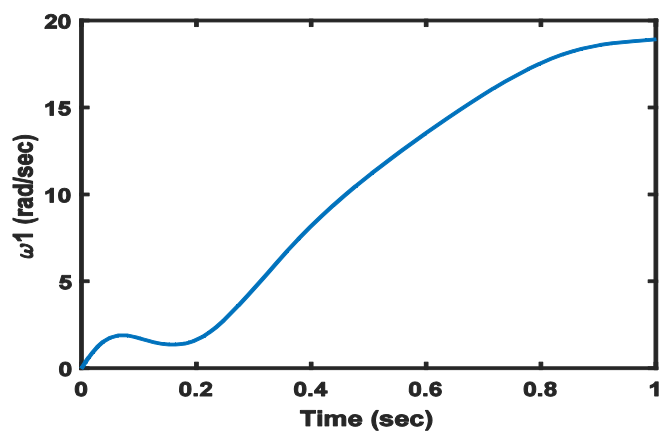

Figure 13. The rotor speed of machine \#1

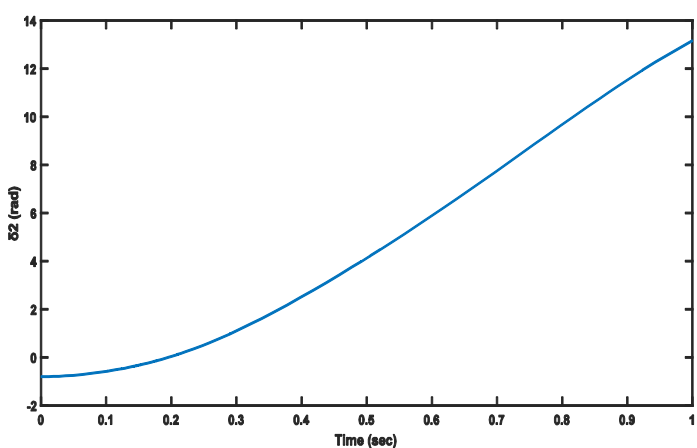

Figure 15. Rotor angle of machine \#2

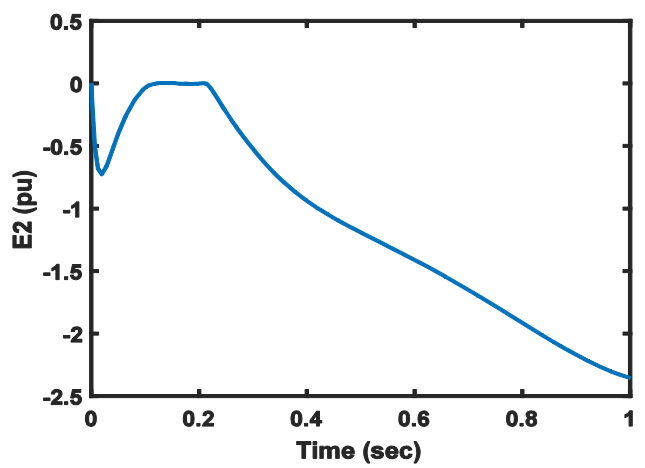

Figure 17. Excitation system of machine \#2 


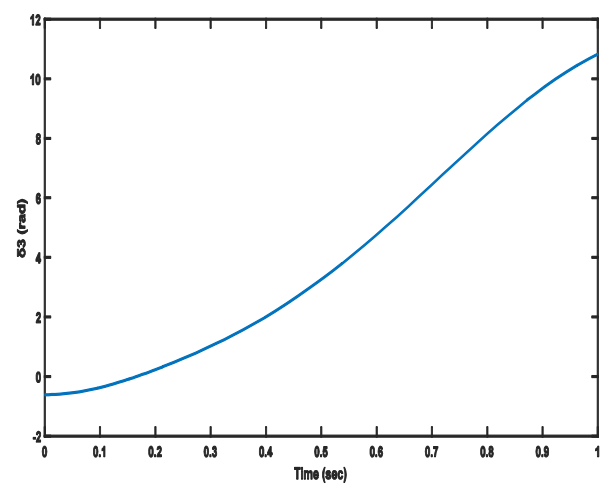

Figure 18. Rotor angle of machine \#3

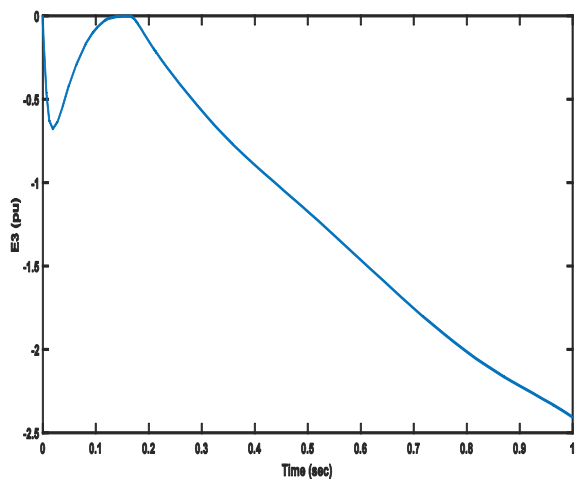

Figure 20. Excitation system of machine \#3

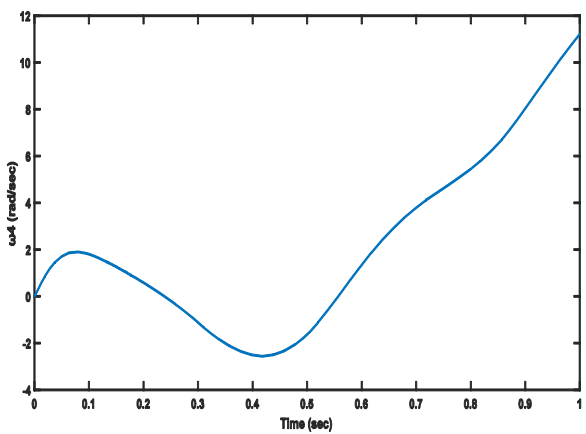

Figure 22. The rotor speed of machine \#4

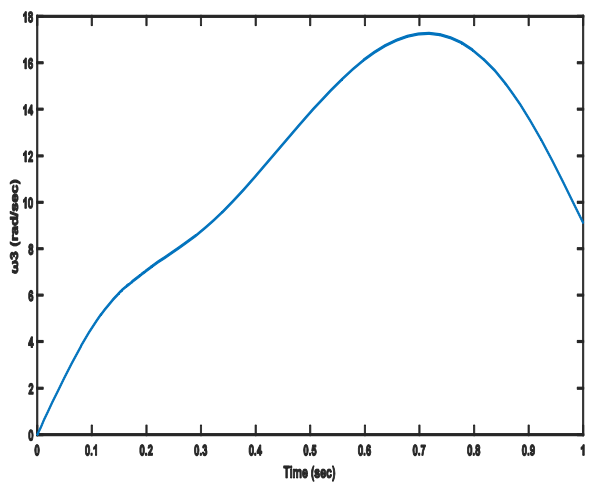

Figure 19. The rotor speed of machine \#3

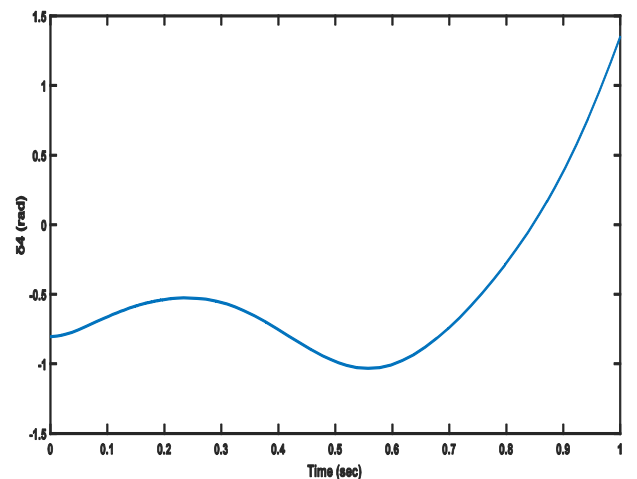

Figure 21. Rotor angle of machine \#4

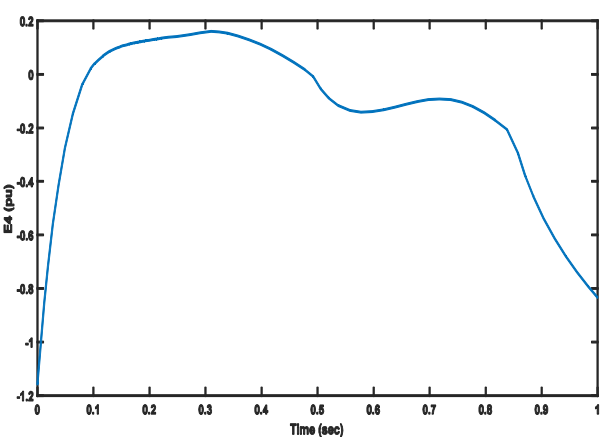

Figure 23. Excitation system of machine \#4

\subsection{Analysis of the Results}

This section analyzes the performance of the closed-loop system. Which are subjects, both to a threephase fault occurs at the transmission line between buses. Simulation of the proposed control structure of the multimachine power system, under this condition, leads to the following observations. It presents the resulting rotor angles versus times; rotor speeds versus times and the excitation system versus times are shown in the figures with corresponding.

The control technique requires feedback from each subsystem. Decentralized through subsystem coordinating a local feedback controller is considerate to communicate among subsystem with constraints 
bounded. The closed-loop system is asymptotically stable with approved performance measures. The advantage of a decentralized controller is reliable and expandable for each subsystem seems to achieve its objective. The decision takes place locally, and negotiation can take place in different actuators. The system exchanges and subproblems can usually be cooperative or competitive.

\section{CONCLUSION}

This paper presents decentralized constrained optimal control to enhance the stability of the multimachine power system. The main contribution and simulation results based on the Algebraic Riccati Equations using the decentralized constrained controller to improve the stability of the multimachine power system has been achieved. Different approaches explore it and compute the following conclusion:

First, the stability of the multimachine power systems for state constraints bounded and all its poles are in the closed left-half plane. Second, the decentralized optimal controller has been proposed, and the feedback from the subsystems is to control themselves.

Improving the stability of the multimachine power systems assumed a lossy transmission line to measure and estimate the values of excitation and angles. Designed optimal controller decentralized with state constraints bounded. Decentralization is an effective way to handle complexity and cross changes in the interaction of large-scale dynamic systems. It often controls the large-scale system by over one controller or decision-maker encompassing decentralized computations.

It satisfies the stability of the overall closed-loop. In any control design, it pursues a controller not to stabilize the system outbuilding also to guarantee the adequate performance of the system. All the subsystems level to secure the entire subsystem asymptotically stable with a performance measure. It has shown that the controller is not aggressive. The paper has also demonstrated that the proposed results yield necessary and sufficient application to the optimal constraint control of three machines and four machine power systems and validate the findings. It has not only stabilized the multimachine power system but also completes the suboptimal control guaranteed, cost performance index for all bounds controlled.

\section{REFERENCES:}

[1] A. A. Fouad and M. A. Paul, Power Systems Control and Stability 2nd Ed, New Jersey: Wiley and Sons, 2008.

[2] J.Machowski, J. B and B. J. R, Power System Dynamic and Stability, Sussex: Wiley, 2008.

[3] J.H.Chow, W. F. F and M. J. A, Applied Mathematics for Restructured Electric Power Systems, New York: Springer, 2005.

[4] Z. Xi, G. F and D. C, "Nonlinear decentralized saturated controller design for power systems," IEEE, vol. 11, no. 4, pp. 539-547, 2003.

[5] R. Ortega, G. Martha, A. Alessandro, S. Yuanzhang and S. Tielong, "Transient stabilization of multimachine power systems with nontrivial transfer conductances," IEEE, vol. 50, no. 1, pp. 60-75, 2005.

[6] H.Li and Y. W, "Nonlinear robust decentralized control of multimachine power systems," IEEE, p. 6, 2005.

[7] A.A.Abouelsoud, I. A.-O. Abdullah and A. Hany, "Observer-Based Stabilizer of A Single Machine Power System with Lossy Transmission Line," WSEAS Transaction On Power Systems, vol. 9, no. 2224-350X, pp. 462-468, 2014.

[8] X.Huang, H. Q, X. Z, L. L and Y. H, "Optimal feedback excitation decentralized coordinated control model of the multimachine power system," Applied mechanics and materials, vol. 433, pp. 1343-1346, 2013.

[9] T.-T. Lee and L. Guang-Tsong, "The inverse problem of linear optimal control for Constance disturbance," International Journal of Control, vol. 43, pp. 233-246, 2007.

[10] A.A.Abouelsoud, "Global stabilization of linear time-invariant system subject to state and control constraints," The Mediterranean Journal of Measurement and control, vol. 3, no. 4, pp. 157-163, 2007.

[11] S.Elloumi, B. A and B. N. Benhadj, "On decentralized control of a multimachine power system: A comparative study," International Journal of Sciences and Technology of Automatic Control and Computer Engineering, vol. 7, no. 1, pp. 1816-1833, 2013.

[12] Z. Hongshan and Lan Xiaoming, "Excitation prediction control of multi-machine power systems using balanced reduced model," in IEEE Power \& Energy Society General Meeting, Vancouver, BC, Canada, 2013.

[13] H. Rtibi and S. Elloumi, "Robust Decentralized Nonlinear Control for Multimachine Power Systems," in International Conference on Advanced Systems and Electric Technologies (IC_ASET), Hammamet, Tunisia, 2017.

[14] T.Bian, Y. J and Z.-P. J, "Decentralized Adaptive Optimal Control of Large Scale Power Systems with Application to Power Systems," IEEE, vol. 62, no. 3, pp. 2439-2447, 2015.

[15] D.Langarica-Cordoba, R. Ortega and D. C, "Transient Stabilization of Multimachine Power Systems: Towards a decentralized global solution," European Journal of Control, p. 26, 2015.

[16] M. Galaz, R. Ortega, A. Astolfi, Y. Sun and T. Shen, "Transient Stabilization of Multimachine Power Systems," Springer, pp. 367-386, 2006.

[17] A.A.Abouelsoud, "Stabilizing output feedback controller of bilinear systems," International Journal Modelling, Identification and Control, vol. 6, no. 4, pp. 313-319, 2009.

[18] K.R.Padiyar, Power System Dynamics: Stability and Control, Hyderabad: BS Publications, 2008. 
[19] Qian.Lu, J. L, K. Gao Jixang, G. K and F. Lee, "Decentralized control for multimachine power systems," Optimal control and application and methods, vol. 10, no. 1, pp. 53-64, 1989.

[20] Q.Lu, S. M, W. H, W. F. F, Y. N and T. Shen, "Nonlinear Decentralized disturbance attenuation excitation control via new recursive design for multimachine power systems," IEEE, vol. 16, no. 4, pp. 729-736, 2001.

[21] Peter.Dorato, A. C. T and V. Cerone, Linear Quadratic Control: An Introduction, New York: KRIEGER, 2000.

[22] M. Menner and N. Z. Melanie, "Convex Formulations and Algebraic Solution for Linear Quadratic Inverse Optimal Control," in European Control Conference (ECC), Limassol, Cyprus, 2018.

[23] H. Setiadi and O. J. Karl, "Power System Design using Firefly Algorithm for Dynamic Stability Enhancement," Indonesian Journal of Electrical Engineering and Computer Science, vol. 1, no. 3, pp. 446-455, 2016.

[24] B. Bentouati, C. Lakhdar and C. Saliha, "Optimal Power Flow using the Moth Flam Optimizer: A case study of the Algerian Power System," Indonesian Journal of Electrical Engineering and Computer Science, vol. 1, no. 3, pp. 431-445, 2016.

[25] D. Harikrishna and V. S. N, "Dynamic Stability Enhancement of Power Systems Using Neural-Network Controlled Static-Compasator," TELKOMNIKA Journal Telecommunication, Computing, Electronics, and Control, vol. 10, no. 1, pp. 9-16, 2012. 